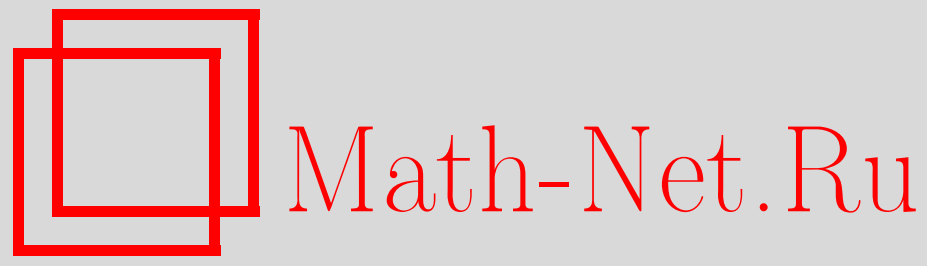

B. А. Ватутин, Рецензия на книгу: Bass Richard F. "Diffusions and Eliliptic Operators", Теория вероятн. и ее примен., 2001, том 46, выпуск 4, 818-820

DOI: https://doi.org/10.4213/tvp3832

Использование Общероссийского математического портала MathNet.Ru подразумевает, что вы прочитали и согласны с пользовательским соглашением

http://www. mathnet.ru/rus/agreement

Параметры загрузки:

IP : 3.95.254.165

26 апреля 2023 г., 13:04:41

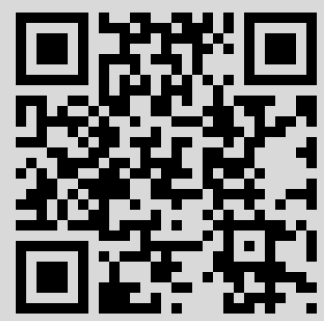


оценивание параметров в распределении экстремального значения.

Глава 7 посвящена линейным временным рядам, шумы которых имеют тяжелые хвосты (введение в теорию временных рядов, авторегрессионные процессы с шумами, не имеющими конечной дисперсии, оценка автокорреляционной функции, периодограммы, задачи оценки параметров для авторегрессии со скользящим средним). В этой главе также затрагиваются нелинейные авторегрессионные модели с тяжелыми хвостами такие, как ARCH-модели.

В гл. 8 обсуждаются специальные предметы такие, как индекс экстремальности, т.е. величина, характеризующая связь между зависимостью и экстремальным поведением в модели, индекс больших выплат, т.е. мера вклада больших выплат в конечную сумму, вопрос о том, когда и как случается разорение в традиционных крамеровских предположениях и в случае больших выплат, бессрочные ренты и ARCH-процессы, свойства самой длительной серии успехов, договоры перестрахования, устойчивые процессы.

В четырех приложениях приведены сведения о типах сходимости (приложение 1), о слабой сходимости в метрических пространствах (приложение 2), о правильном изменении и субэкспоненциальности (приложение 3 ) и сведения из теории восстановления (приложение 4).

Книга содержит обширный и удобный в использовании вспомогательный материал: список литературы содержит 646 наименований, в алфавитный указатель входит более 1000 терминов.

Читатель, желающий не только изучить теорию экстремальных событий, но и найти иллюстрации ее применения, например, на основе величин 4580 страховых выплат по портфелю страхования от огня или данньх о ежедневной логарифмической доходности по акциям компании БМВ за период с 2 января 1973 г. по 23 июля 1996 г., с интересом ознакомится с этой книгой.

\section{II.2001 г.}

В. К. Малиновский

B a s s R i c h a r d F. Diffusions and Elliptic Operators. Probability and Applications. New York, Berlin, Heidelberg: Springer-Verlag, 1998, xiv+232 p.

Одним из интересных направлений развития современньг математических методов является взаимопроникновение и взаимообогащение теории вероятностей и теории дифференциальньх уравнений. Надо сказать, что эта тематика нашла довольно широкое отражение в литературе. Достаточно вспомнить как книги [1]-[3], вышедшие довольно давно и ставшие уже классическими, так и монографии [4]-[5], появившиеся в последние годы. Однако эти издания предназначены, как правило, тем читателям, которые имеют достаточно глубокие знания в теории вероятностей или, если быть более точным, в теории стохастических дифференциальных уравнений и теории мартингалов. Рецензируемая книга имеет несколько иную направленность. Ее цель ознакомить широкий круг читателей, в том числе не имеющих специального вероятностного образования, с методами теории вероятностей, которые могут оказаться полезными при исследовании свойств решений различньх классов уравнений в част-

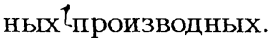

Остановимся на содержании книги более подробно.

Первая глава «Стохастические дифференциальные уравнения» имеет вводный характер и содержит основные сведения из теории вероятностей: определения мартингала, локального мартингала, броуновского движения, стохастического дифференциального уравнения (для случая $n$ переменньх), теорему Гирсанова, определение и простейшие свойства интеграла Стратоновича, потраекторные свойства стохастических дифференциальных уравнений.

Вторая глава «Представления решений» является, по сути дела, оправданием к написанию книги. Прочитав ее, даже неискушенный читатель сможет оценить мощь 
и возможности вероятностных идей. Основное внимание в этой главе, впрочем, как и в последующих, уделяется уравнениям, включающим в себя операторы $\mathscr{L}$ вида

$$
\mathscr{L} f(\mathbf{x})=\frac{1}{2} \sum_{i, j=1}^{d} a_{i j}(\mathbf{x}) \frac{\partial^{2} f(\mathbf{x})}{\partial x_{i} \partial x_{j}}+\sum_{i=1}^{d} b_{i}(\mathbf{x}) \frac{\partial f(\mathbf{x})}{\partial x_{i}}, \quad \mathbf{x} \in \mathbf{R}^{d}
$$

где

$$
\sum_{i, j=1}^{d} a_{i j}(\mathbf{x}) y_{i} y_{j} \geqslant \Lambda(\mathbf{x}) \sum_{i=1}^{d} y_{i}^{2}
$$

для некоторой функции $\Lambda(\mathbf{x})$ при всех $\left(y_{1}, \ldots, y_{d}\right) \in \mathbf{R}^{d}$.

Не касаясь проблем существования и единственности, а заранее предполагая, что решения рассматриваемьх уравнений существуют и обладают определенной степенью гладкости, автор дает вероятностные представления в терминах математических ожиданий функционалов от диффузионных процессов для решений уравнения Пуассона в ограниченной и неограниченной областях, уравнения Шредингера, задач Дирихле, Коши, Неймана, а также обсуждает вопрос существования фундаментального решения для сопряженньх операторов.

В главе III «Регулярность решений» при помощи метода возмущений доказывается, что упомянутые выше уравнения имеют решения, обладающие необходимой степенью гладкости, и тем самым устанавливается, что вероятностные представления предыдущей главы для решений этих уравнений действительно справедливы.

В главе IV «Одномерные диффузионные процессы» освещены вопросы, относящиеся к естественной временной шкале регулярных диффузионньх процессов, в частности, указаны методы нахождения естественной шкалы в терминах решений дифференциальньх уравнений, связанных с соответствующими диффузионными процессами.

В главе $\mathrm{V}$ «Операторы недивергентного вида» рассматриваются равномерно эллиптические диффузионные операторы вида (1), удовлетворяющие дополнительным условиям: $\sup _{i}\left\|b_{i}\right\|_{\infty} \leqslant \Lambda_{1}$,

$$
\Lambda_{2} \sum_{i=1}^{d} y_{i}^{2} \leq \sum_{i, j=1}^{d} a_{i j}(\mathbf{x}) y_{i} y_{j} \leq \Lambda_{2}^{-1} \sum_{i=1}^{d} y_{i}^{2}, \quad \mathbf{x} \in \mathbf{R}^{d}, \mathbf{y} \in \mathbf{R}^{d}
$$

для некоторых констант $\Lambda_{1}, \Lambda_{2}$. Здесь особое внимание уделено теореме Крылова об аппроксимации резольвент и версии Крылова-Санова неравенства Харнака для положительных $\mathscr{L}$-гармонических функций.

В главе VI «Мартингальные проблемы» рассматриваются вопросы существования и единственности решения мартингальной проблемы для равномерно эллиптических диффузионных операторов $\mathscr{L}$.

В главе VII «Операторы дивергентного вида» изучаются равномерно эллиптические диффузионные операторы, удовлетворяющие условию (3) и имеющие вид

$$
\mathscr{L} f(\mathbf{x})=\frac{1}{2} \sum_{i, j=1}^{d} \frac{\partial}{\partial x_{i}}\left(a_{i j}(\mathbf{x}) \frac{\partial f(\mathbf{x})}{\partial x_{j}}\right),
$$

где $a_{i j}(\mathbf{x})=a_{j i}(\mathbf{x})$ для всех $i, j$ и $\mathbf{x}$. Понятно, что если функции $a_{i j}(\mathbf{x})$ являются гладкими, то указанные операторы являются частными случаями операторов, исследовавшихся в главе V. Однако специфическая форма операторов (4) позволяет получать оценки сверху и снизу для переходных плотностей диффузионньг процессов, соответствующих таким операторам, и без предположений о гладкости функций $a_{i j}$. Именно этим вопросам и посвящена глава VII.

Заключительная глава носит название «Исчисление Малливена». Эта тема заслуживает по крайней мере монографии. Поэтому автор концентрируется лишь 
на применении исчисления Малливена к доказательству гладкости по у переходных плотностей $p(t, \mathbf{x}, \mathbf{y})$ процессов, соответствующих операторам $\mathscr{L}$ с гладкими коэффициентами. Существуют два основных подхода к решению этой задачи: метод, опирающийся на теорему Гирсанова, и метод, использующий оператор ОрштейнаУленбека. Оба метода достаточно подробно обсуждаются в рассматриваемой главе.

Конечно в книге учебного характера трудно охватить все аспекты взаимообогащения теории вероятностей и теории дифференциальных уравнений в частньх производньг, но изучив ее, читатель несомненно будет подготовлен к чтению более сложных книг, в той или иной мере касающихся данной области.

20.I.2000 г.

B. A. Bamyтuн

\section{СПИСОК ЛИТЕРАТУРЫ}

1. Гихман И.И., Скороход А.В. Стохастические дифференциальные уравнения. Киев: Наукова думка, 1968, 354 с.

2. Stroock D. W., Varadhan S.R.S. Multidimensional Diffusion Processes. New York: Springer-Verlag, 1979, $338 \mathrm{p}$.

3. Ватанабе ЩЦ., Икэда Н. Стохастические дифференциальные уравнения и диффузионные процессы. М.: Наука, 1986, 448 с.

4. Dawson D. A., Perkins E. A. Historical Processes. - Mem. Amer. Math. Soc., 1991, v. 93, № 454, 179 p.

5. Dynkin E. B. An Introduction to Measure-Valued Processes. CRM Monographs Series, 6, Providence: Amer. Math. Soc., 1994, 134 p. 